
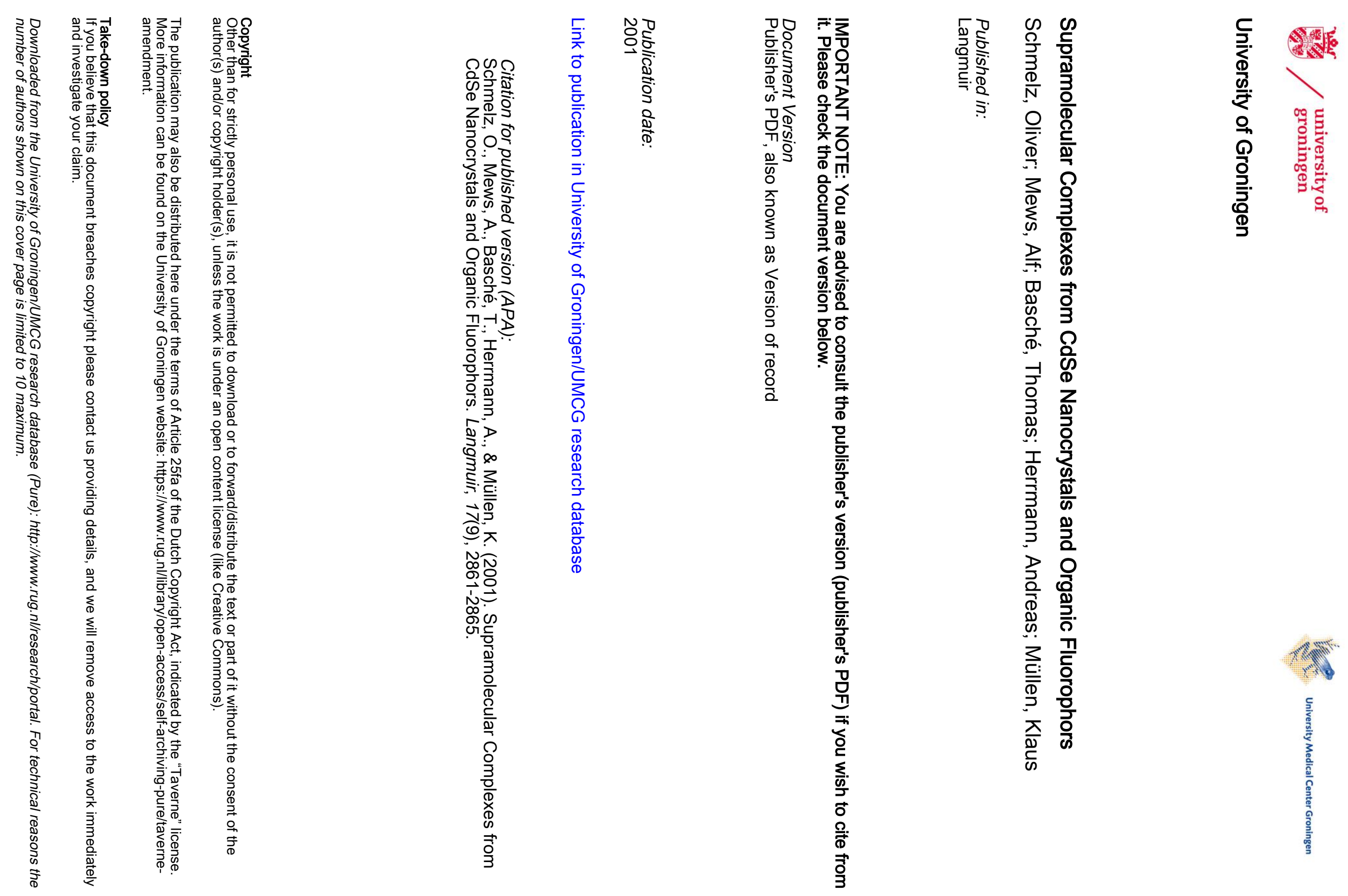


\title{
Supramolecular Complexes from CdSe Nanocrystals and Organic Fluorophors
}

\author{
Oliver Schmelz, ${ }^{\dagger}$ Alf Mews, ${ }^{*}{ }^{\dagger}$ Thomas Basché, ${ }^{\dagger}$ Andreas Herrmann, ${ }^{\ddagger}$ and \\ Klaus Müllen ${ }^{\ddagger}$ \\ Institut für Physikalische Chemie, Wélderweg 11, 55099Mainz, Germany, and \\ Max-Planck-Institut für Polymerforschung, Ackermannweg 1, 55128Mainz, Germany
}

Received November 27, 2000. In Final Form: February 14, 2001

\begin{abstract}
Heterosupramolecular structures from $\mathrm{d} \mathrm{dS}$ e or $\mathrm{CdSe} / \mathrm{ZnS}$ core/shell nanocrystals and a specially designed organic fluorophore (diazaperylene) have been prepared to investigate the electronic interactions at nanoscopic semiconductor surfaces. To determine the average number of dye molecules per nanocrystal, wemonitored thesize-dependent molar extinction coefficient of $\mathrm{C} d S$ eparticles. The NC /dyeratiois depending on the particle size and varies between 3 and 20 dye molecules per nanocrystal. We demonstrate that the fluorescence of the nanocrystals is completely quenched upon the formation of the supramolecular NC/dye complex even if the surface of the CdSe particles is covered with a few monolayers of the high band gap semiconductor $\mathrm{ZnS}$.
\end{abstract}

\section{Introduction}

The use of semiconductor nanocrystals (NCs) in nanotechnol ogy strongly depends on the ability toattach these particles tospecificlocations of potential devices. Colloidal particles prepared by wet chemical methods provide this flexibility because they are covered with molecular ligands. The ligands can serve as molecular wires as has been shown for nanocrystals bound between nanoelectrodes via bifunctional dithiols wheresingle-electron charging effects could be observed. ${ }^{1,2}$ Another interesting application of semiconductor nanocrystals is their use in biolabeling. It was demonstrated that the particles can be attached to appropriate binding sites in biological systems where the size-dependent color variation of the NCs was used for multicolor fluorescence imaging ${ }^{3}$ However, the nature of the ligands can influence the photophysics of the particles tremendously. ${ }^{4}$ Especially the nanocrystal fluo rescence can be quenched because certain ligands may serveas scavengers for the photogenerated charge carriers. Therefore inorganic surface passivation, i.e., overcoating of the particles with a material of higher band gap, has been utilized. ${ }^{5}$ In the overcoated particles the charge carriers are mainly localized in the core which increases the fluorescence quantum yield to more than $50 \%{ }^{6,7}$ In the present work weinvestigate theelectronicinteraction of the overcoated particles with their molecular surface ligands in detail. To monitor the electronic properties of the particles and the ligands by fluorescence measure ments, we have used a new kind of dye molecule as a

* To whom correspondence may be addressed. Phone: ++ 49 6131 3922982. Fax ++ 496131 3923953. E-mail: alf.mews@ uni-mainz. de.

${ }^{\dagger}$ Institut für Physikalische Chemie.

‡ Max-Planck-Institut für Polymerforschung.

(1) Klein, D. L.; Roth, R.; Lim, A. K. L.; Alivisatos, A. P.; McEuen, P. L. Nature 1997, 389699.

(2) Banin, U.; Cao, Y. W.; Katz, D.; Millo, O. Nature 1999, 400542

(3) Bruchez, M.; Maronne, M.; Gin, P.; Weiss, S.; Alivisatos, A. P. Science 1998, 2812013

(4) Kuno, M.; Lee, J. K.; Dabbousi, B. O.; Mikulec, F. V.; Bawendi, M. G. J. Chem. Phys. 1997, 1069869.

(5) Hässelbarth, A.; Eychmüller, A.; E ichberger, R.; Giersig, M.; Mews, A.; Weller, H. J. Phys. Chem. 1993 97, 5333.

(6) Hines, M. A.; Guyot-Sionnest, P. J. Phys. Chem. 1996100468.

(7) Peng, X. G.; Schlamp, M. C.; Kadavanich, A. V.; Alivisatos, A. P. J. Am. Chem. Soc. 1997, 1197019. functional ligand. The specially designed diazaperylene dye is a perylene derivate with twonitrogen atoms at one of the bay areas which serve as specific binding sites to thenanocrystal surface. TheNC/dyeratiowas determined from the size-dependent molar extinction coefficient of the nanocrystals. These "heterosupramolecular" complexes $^{8}$ are considered to be prototype structures for electronic interactions at nanoscopic semiconductor surfaces the understanding of which is of great interest for, e.g, photovoltaic devices based on nanoparticles. ${ }^{9-11}$

\section{Results}

In the last years $\mathrm{C} d \mathrm{dSe}$ particles have become a model system in nanocrystal research because their band gap can be tuned over almost the whole visible range and the particles can besynthesized with a remarkablesizecontrol and very high crystallinity. ${ }^{12}$ The upper part of $\mathrm{F}$ igure 1 shows absorption spectra of $\mathrm{CdSe}$ particles of different sizes which were prepared via high-temperature organometallic chemistry. ${ }^{13,14}$ B riefly, a solution of dimethylcadmium and selenium powder in tributlyphophine (TBP) was injected in hot trioctylphosphineoxide (TOPO) $\left(T>300^{\circ} \mathrm{C}\right)$ whereby the desired particlesizewas achieved by varying thetemperature, theconcentration of reactants, and the reaction time. The overcoating of the particles with $\mathrm{ZnS}$ was performed according to ref 15 ; i.e., CdSe particles were dissolved in molten TOPO and the $\mathrm{ZnS}$ shell was grown by addition of diethylzinc and hexamethyldisilathiane $\left((\mathrm{TMS})_{2} \mathrm{~S}\right)$.

To use these particles as chemical precursors to build up more complex structures, like NC /dye composites, the particle concentration in solution needs to be known. In general, theconcentration could be determinedvery easily

(8) Cusack, L.; Rao, N.; Fitzmaurice, D. Chem. Eur. J. 1997, 3202 (9) O’Regan, B.; Grätzel, M. Nature 1991, 353737.

(10) Vogel, R.; Pohl, K.; Weller, H. Chem. Phys. Lett. 1990174241.

(11) Ginger, D. S.; Greenham, N. Phys. Rev. B 1999, 59, 10622.

(12) Alivisatos, A. P. J. Phys. Chem. 199610013226

(13) Murray, C. B.; Norris, D. J.; Bawendi, M. G. J. Am. Chem. Soc. 1993, 1158706

(14) Bowen Katari, J. E.; Colvin, V. L.; Alivisatos, A. P. J. Phys. Chem. 1994984190.

(15) Daboussi, B. O.; Rodriguez-Veijo, J.; Miculec, F. V.; Heine, J. R. Matoussi, H.; Ober, R.; Jensen, K. F.; Bawendi, M. G. J. Phys. Chem. B 1997, 1O1 9436. 

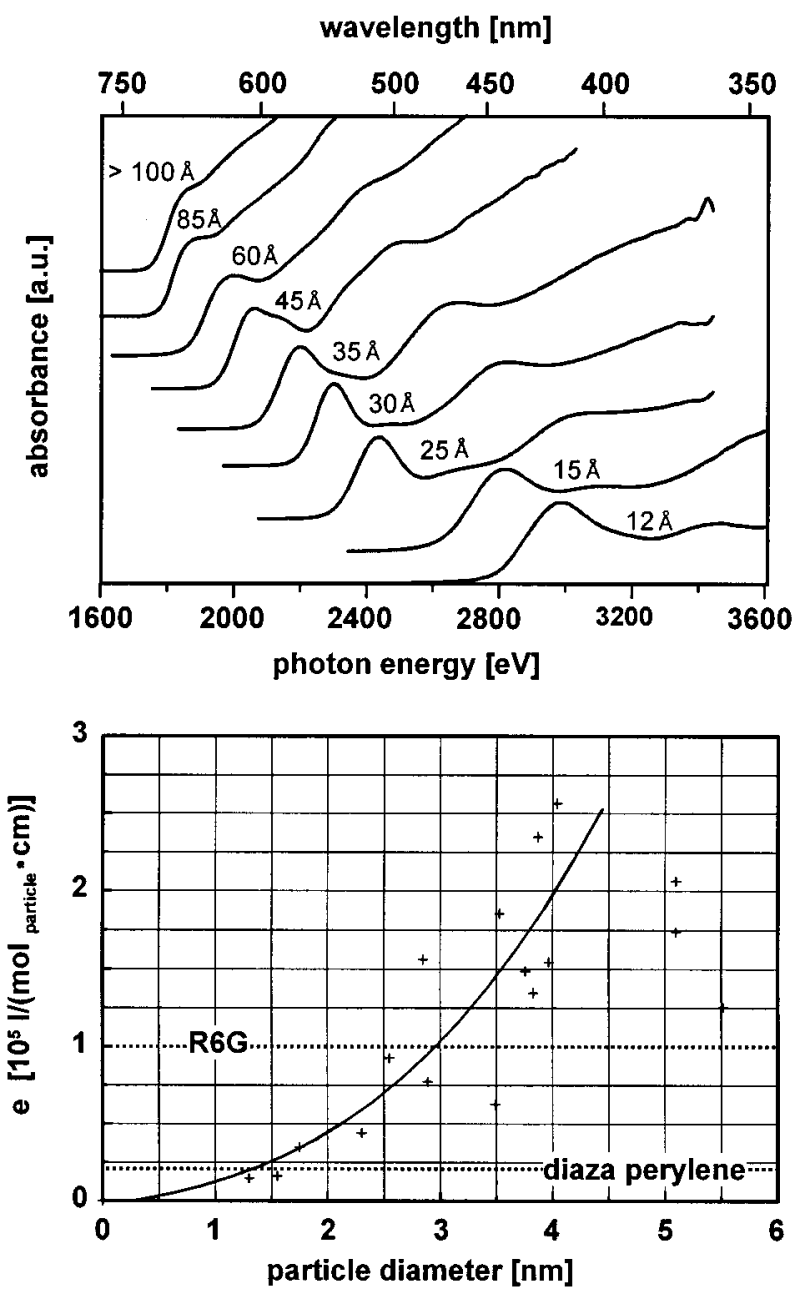

Figure 1. The upper graph shows a set of $\mathrm{CdSe}$ nanocrystals in a sizeregime of $1-10 \mathrm{~nm}$ in diameter. Thelower graph shows their molar extinction coefficients at their first absorption maximum Particles $3 \mathrm{~nm}$ in diameter (250 CdSe units) have the same extinction coefficient as R6G. The molar extinction coefficient of the diazaperyleneis $1.8 \times 10^{4} \mathrm{~L} /(\mathrm{mol} \mathrm{cm})$. Deviation from the aubic dependence of the absorption coefficient upon particle diameter (solid line) may be due to different size distribution or overlapping absorption bands.

by optical absorption measurements if their molar extinction coefficient would be known. Although $\mathrm{CdSe}$ nanoarystals were investigated in detail in the past few years, very little is known about their absorption strength. Therefore, we synthesized a set of $\mathrm{CdS}$ e particles and determined the molar extinction coefficient at their first absorption maximum, i.e., at their "excitonic" or "band gap" absorption. Thetotal cadmium concentration of each samplewas determined by atomicabsorption spectroscopy (AAS) after digesting the particles in $\mathrm{HNO}_{3}$. The particle concentration was then calculated by assuming bulk lattice parameters and spherical symmetry where the particle diameter was deduced from the absorption spectra. The correspondence of particle diameter andabsorption spectra was taken from the literature. ${ }^{13,14}$ The lower graph in Figure 1 shows thedependence of theabsorption coefficient upon particlediameter. E ven though thereis a fairly large dispersion in the data, which is duetoseveral experimental uncertainties such as variation in size and shape, it can be seen that the trend follows a cubic dependence (solid line) up to a diameter of approximately $4.5 \mathrm{~nm}$; i.e, it appears to scale with the volume of the particles. This observation shows that the absorption coefficient is proportional to the number of $\mathrm{CdSe}$ units in the nano- crystal. In contrast, measurements on colloidal $\mathrm{CdS}$ particles prepared in aqueous solution showed that the oscillator strength is not depending on particle diameter if the size of the nanocrystals is smaller than the bulk exciton. ${ }^{16}$ Above a diameter of approximately $5 \mathrm{~nm}$ the absorption peaks cannot beclearly resolved in thespectra (see upper part of Figure 1). Also even slight variations in size can already lead toa large divergence in molecular weight. Therefore, we found that a reliable estimate of the molar extinction coefficient on the basis of ensemble absorption spectra can only be given up to a diameter of $5 \mathrm{~nm}$ for $\mathrm{CdS}$ e nanocrystals prepared by the described method. By comparison of theabsorption strength tothose of common dye molecules, we have found that $\mathrm{CdSe}$ particles of $3 \mathrm{~nm}$ diameter containing about $250 \mathrm{CdSe}$ units have approximately the same molar extinction coefficient as Rhodamine 6G $\left(1 \times 10^{5} \mathrm{~L} /(\mathrm{mol} \mathrm{cm})\right)$ at the first absorption maximum. The diazaperylene dye which is eventually attached to the nanocrystal surface (see below) has a molar extinction coefficient of $1.8 \times 10^{4} \mathrm{~L} /(\mathrm{mol}$ $\mathrm{cm})$, i.e., comparable to particles with a diameter of 1.5 $\mathrm{nm}$. Therefore, the determination of the size-dependent extinction coefficient allows for a profound estimation of the $\mathrm{NC} /$ dye ratio in the composite structures.

The formation of the $\mathrm{NC} /$ dye composites occurs via a ligand exchange reaction. As mentioned above the synthesis of the particles proceeds in TOPO where the oxygen is attached tothesurfaceand thelongalkyl chains provide solubility in nonpolar solvents such as toluene or hexane. Polar solvents such as methanol or ethand cause precipitation of the particles from solution. ${ }^{13}$ We have investigatedalargenumber of nitrogen-containingligands such as aniline, benzylamine, phenylethylamine, dodecylamine, pyridine, and phenazin compounds. F or macroscopic $\mathrm{CdS}$ e surfaces, it was shown that TOPO and amine ligands are not strongly bound to the surface but that there is a dynamic exchange equilibrium between attached ligands and ligands in solution. ${ }^{17,18} \mathrm{~F}$ or the $\mathrm{CdS}$ e nanocrystals we found that this equilibrium is depending not only on the ligand itself but also on parameters such as particle size, solvent, temperature, andconcentrations. To investigate the affinity of different amines to the nanocrystal surface, the TOPO ligands were first exchanged by pyridine. This was done by subsequently refluxing the particles in pyridine and precipitation through addition of hexanefor several times. The pyridine on the surface of the particles was then exchanged by addition of different amines as could be monitored by NMR spectroscopy. As a general trend in binding strength among the investigated amines, it can be claimed that aromatic amines are less strongly bound to the surface than aliphatic amines where the binding strength increases with the aliphatic chain length. The strongest bonds, however, were formed with bidental ligands such as bipyridyl compounds, a trend which has also been observed at bulk $\mathrm{C} d \mathrm{~S}$ e surfaces. ${ }^{18}$

In view of the strong complexing nature of bipyridyl ligands, ${ }^{19}$ we have synthesized a polyaromatic dye where the bipyridyl groupis involvedin the hromophoricsystem We choosetosynthesize the molecule 1,12-diazaperylene, which contains two nitrogen atoms at one of the two bay regions of the conjugated system as displayed in Scheme

(16) Vossmeyer, T.; Katsikas, L.; Giersig, M.; Popovic, I. G.; Diesner, K.; Chemseddine, A.; Eychmüller, A.; Weller, H. J. Phys. Chem. 1994 987665.

(17) Lorenz, J. K.; Ellis, A. B. J. Am. Chem. Soc. 199812010970.

(18) Lisensky, G. C.; Penn, R. L.; Murphy, C. J.; Ellis, A. B. Science 1990, 248840

(19) Meyer, T. J. Acc. Chem. Res. 1989, 22, 163 
Scheme 1
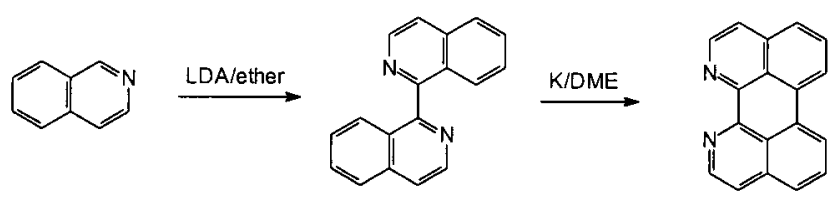

1. Sofar only the synthesis of 1,7- and 3,9-diazaperylene derivatives has been described in the literature ${ }^{20-22}$ whereas 1,12-diazaperylene has only been the subject of abinitioand HMO calculations. ${ }^{23}$ The two-stepsynthesis of 1,12-diazaperylene starts from isoquinoline which is dimerized with LDA in ether toform 1, 1'-biisoquinoline. ${ }^{24}$ The cyclization reaction, which yields 1,12-diazaperylene, was inspired by the observation of Solodovnikov et al. who showed that 1,1 '-binaphthyl spontaneously cyclizes through reduction withalkali metals. ${ }^{25} \mathrm{~F}$ or this particular anionic cyclization reaction, potassium in dimethoxyethane was used.

It turns out that diazaperylene can readily exchange TOPO from the surface of $\mathrm{C} d \mathrm{dS}$ e nanocrystals. This can be seen by investigation of the reaction product of semiconductor nanocrystals and chromophores, which has a very different solubility than the parent compounds. Whereas both isolated compounds are well soluble in toluene, the NC/dye complex, which is immediately formed after addition of excess dyesolution tothecolloi dal nanocrystals at room temperature, precipitates right away. After separation, this product can be washed with toluene and redissolvedin more polar solvents such as dichloroethane. The change in polarity of the prepared heterostructure as compared to the isolated compounds might be a result of charge separation upon attachment. The synthesis of the $\mathrm{NC}$ /dye complex and the corresponding NMR spectrumis shown in Figure 2. It can clearly be seen that the signals of the aromatic protons show strong broadening upon attachment tothe nanocrystal surface and partially shift to lower field. There might be several reasons for the observed broadening such as, e.g, reduced rotational freedom, a variety of different microenvironments, or different orientations of the attached molecules with respect to the nanocrystal surface. However, the same experiments with pure perylene chromophores instead of diazaperylene did not show any of the effects mentioned above. Therefore, theabsence of narrow peaks in theNMR spectra of the diazaperyleneNC solution together with the change of solubility is a strong argument that the formation of a NC/dye complex has taken place.

The strongest argument for the formation, however, is the change in the photophysics of the $\mathrm{NC}$ /dye heterostructure because the fluorescence of the nanocrystals is completely quenched upon attachment of the dye moleaules. Interestingly, the quenching occurs for $\mathrm{CdSe}$ as well as $\mathrm{CdSe} / \mathrm{ZnS}$ nanocrystals. For comparison, theoptical spectra of theisolated compounds are shown in the upper graph of Figure 3. The solid lines show absorption and emission spectra of the diazaperylene in toluene. The lowest absorption band is centered at $442 \mathrm{~nm}$ and the emission is Stokes shifted by $10 \mathrm{~nm}$. The dotted lines show

(20) Naumann, C.; Langhals, H. Chem. Ber. 19901231881

(21) Tatke, D. R.; Seshadri, S. Indian J. Chem., Sect. B 1983 1197. Chem. Abstr. 1984 101 54892g.

(22) Tatke, D. R.; Seshadri, S. Dyes Pigm. 1986, 7,153. Chem. Abstr. $1986104188145 \mathrm{u}$.

(23) Polansky, O. E.; Zander, Motoc, M., I. Z. Naturforsch. 1983, 38, 196.

(24) Ashby, M. T.; Govindan, G. N.; Grafton, A. K. J. Am. Chem. Soc. $1994,116,4801$.

(25) Solodovnikov, S. P.; Ioffe, Yu, S. T.; Zaks, B.; Kabachnik, M. I. Bull. Acad. Sci. USSR, Div. Chem. Sci. (Engl. Transl.), 1968, 442 the absorption and emission spectra of $\mathrm{C} d$ S enanocrystals, which are about $3 \mathrm{~nm}$ in diameter and covered with nominally onetotwomonolayers of $\mathrm{ZnS}$. These particular nanocrystals have their first absorption band at $550 \mathrm{~nm}$ and exhibit a fluorescence quantum yield of more that $50 \%$ as compared to a common dye molecule (R6G) emitting at a similar wavelength.

The lower graph in Figure 3 shows optical spectra of the NC(CdSe/ZnS)-dye complex (solid lines) and the absorption of the pure nanocrystals for comparison (dotted line). F rom the extinction coefficients it can be estimated that the NC/dye ratio for this particular sample is $1 / \mathrm{B}$. This value changes for bigger-sized nanocrystals where ratios of up to 20 dye molecules per nanocrystal were observed. We found that the absorption spectrum (abs.) of the NC/dye complex is not a pure superposition of the spectra for isolated dyes and nanocrystals. Instead, it shows an additional band at $474 \mathrm{~nm}$, the origin of which is not absolutely clear yet. This band appears for any NC / dye complex investigated and depends neither on the nanocrystal size nor on the $\mathrm{ZnS}$ shell thickness.

A very interesting point tonotice is that we could never detect nanocrystal fluorescence for any sample investigated after the dyes were attached to the surface. The inset in the lower graph of Figure 3 shows a fluorescence spectrum of the NC/dye complex upon excitation at 390 $\mathrm{nm}$. This spectrum is quite similar to the fluorescence spectrum of the pure dye and shows no evidence of NC fluorescence. In addition the fluorescence excitation spectrum which was recorded at a detection wavelength of $590 \mathrm{~nm}$, i.e., at a wavelength where mostly NC fluorescence is expected, only shows the features of the dye absorption including the extra band at $475 \mathrm{~nm}$. The
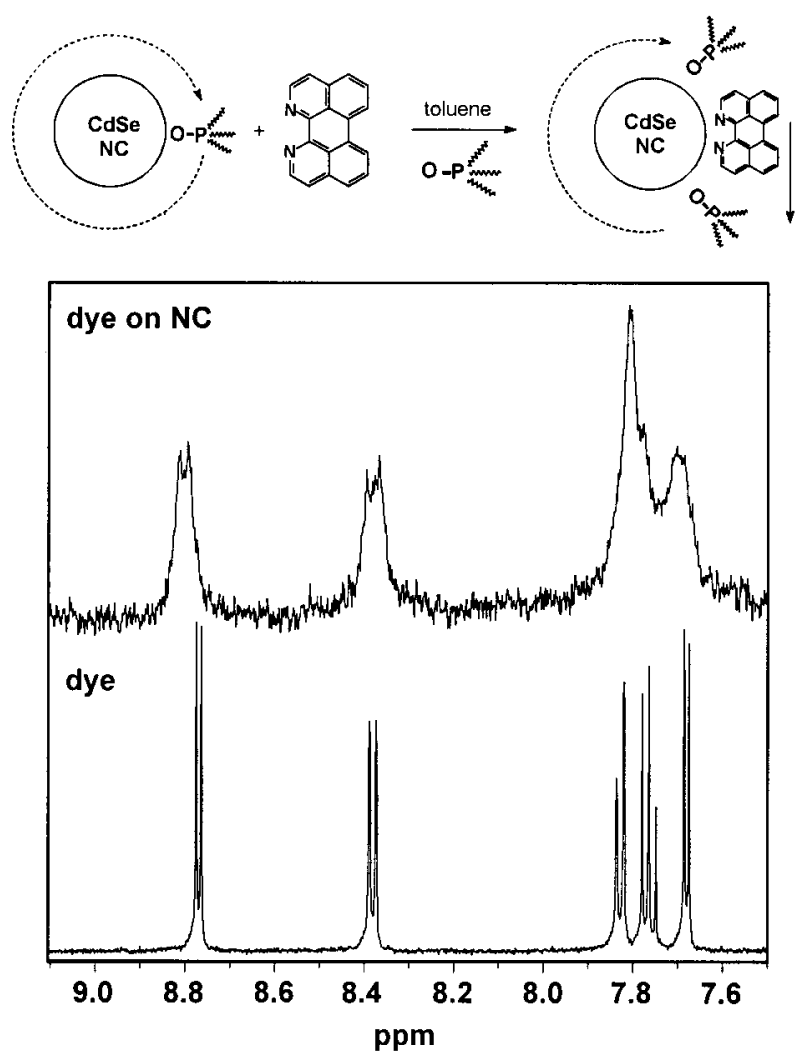

Figure 2 Scheme for the synthesis of nanocrystal-dye complexes and corresponding NMR spectra. Whileboth isolated compounds are readily solublein toluene, the NC-dyecomplex precipitates and can beredissolvedin, e.g. chloroform. TheNMR signals (in $\mathrm{CD}_{2} \mathrm{Cl}_{2}$ ) of the dye show massive broadening upon attachment of the dye to the NC surface. 


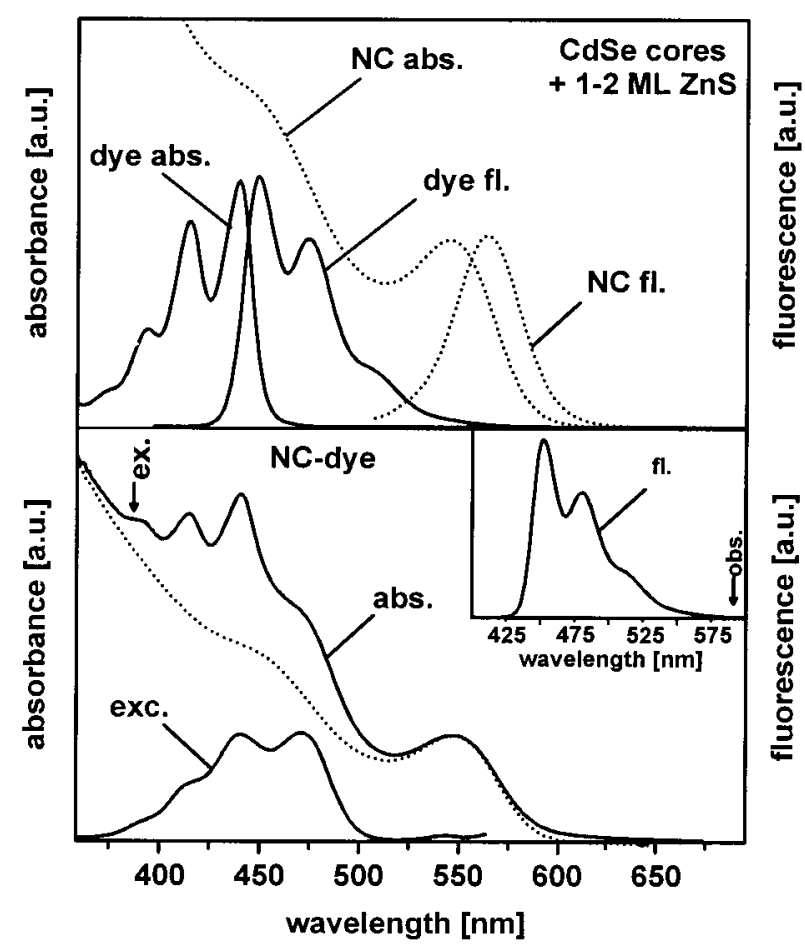

Figure 3 Fluorescence and absorption spectra of isolated compounds (top) and of NC/dye complex (bottom). The fluo rescence and absorption spectra of the dye are almost mirror images while thenanocrystals showincreasing absorption above the "band gap" and a symmetric fluorescence band (quantum yield approximately $50 \%$ for $\mathrm{ZnS}$ covered $\mathrm{CdS}$ e particles). The absorption spectrum of the NC/dye complex (abs.) is not a pure superposition of both spectra but shows an additional band at $470 \mathrm{~nm}$. The inset in the lower graph shows the fluorescence spectrum of the NC /dye complex (fl.), which is very similar to that of the pure dye. As the fluorescence excitation spectrum (exc) recorded at $590 \mathrm{~nm}$ reveals almost only dye absorption, the nanocrystal fluorescence is completely quenched. The fluorescence quenching is alsoobserved in a wavelength region (500-600 nm) when only the nanocrystals areexcited (for details see text).

absence of the absorption features of the nanocrystal suggests that the NC excited state is actively quenched by the dye even when the NC/dye compound is excited below the absorption threshold of the dye, i.e., between 500 and $600 \mathrm{~nm}$ where only nanocrystal absorption takes place.

\section{Discussion}

The origin of the additional band at $474 \mathrm{~nm}$ is not absolutely clear yet. There might be several reasons for this band, such as, e.g., the formation of interfacial electronic states upon attachment of the dye molecules to the nanocrystal surface or a change of the molecular environment upon attachment. To get a qualitative measure of possible environmental effects, we recorded absorption spectra of pure diazaperylene which was blended into a polymer or spin cast on a glass cover slip. For both samples we could observe a red shift of the absorption spectrum by about $30 \mathrm{~nm}$. Therefore thechange of the dye spectrum upon attachment might not reflect electronic interactions with the NCs such as a chargetransfer band. It might rather be due to different microenvironments which in turn lead to different classes of adsorbed dye molecules with variations in their absorption spectra. Another possibility for the change of the dye absorption spectra would be the formation of dye aggregates. ${ }^{26}$ Additional theoretical calculations on the

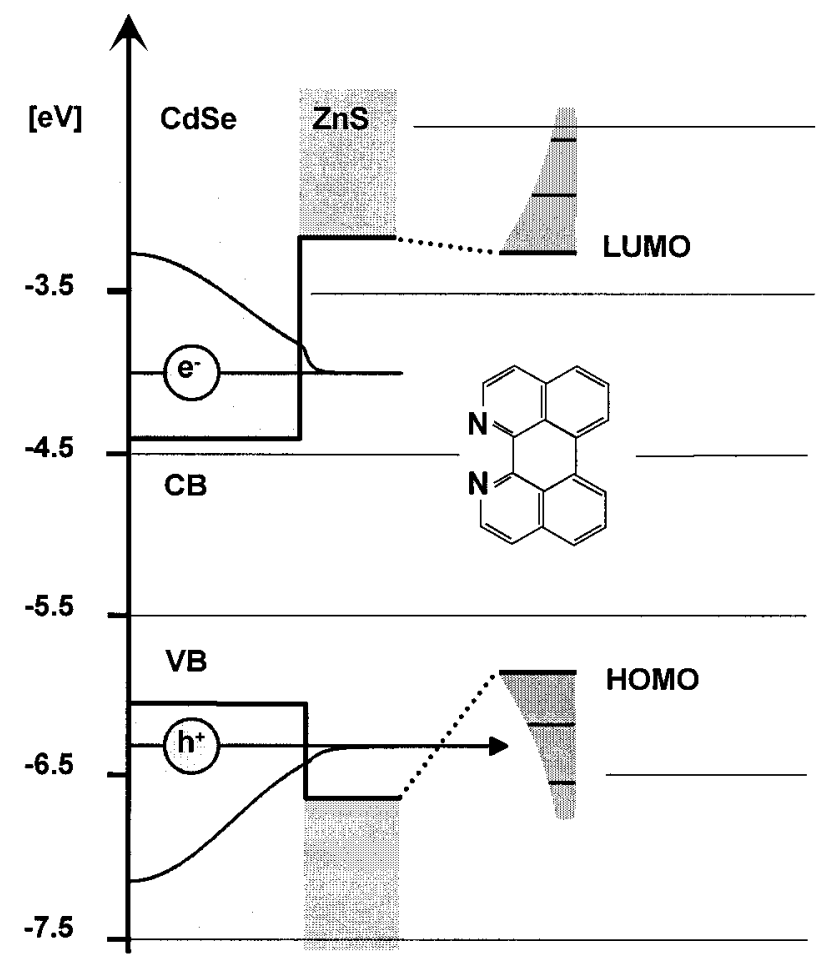

Figure 4. Electroniclevel scheme of the NC/dyecomplex. The shaded areas on the left-hand side show the conduction and valence bands of $\mathrm{CdSe}$ and $\mathrm{ZnS}$, inside of which the quantized electron and hole levels and their wave functions are drawn. The right side shows the absolute positions of the HOMO and LUMO of diazaperylene as determined by $\mathrm{CV}$ measurements. The fact that the nanocrystal fluorescence is also quenched if only the NC and not the dye is excited suggests a hole transfer from thenanocrystal valenceband totheHOMO of theattached dye molecule (for details see text).

electronic level structure of the diazaperylene dye and dye aggregates as well as their electronicinteraction with semiconductor surfaces are needed to explain the absorption spectra of the NC/dye compound in all detail.

Theeffect of fluorescence quenching of the nanocrystals upon attachment of the dye molecules can be discussed by means of an electronic level structure that is shown in Figure 4. The shaded areas on the left side of the picture show the conduction and valence bands of macroscopic $\mathrm{CdS}$ e and $\mathrm{ZnS}$ in which the quantized electron and hole wave functions are drawn, respectively. The energetic positions of the $\mathrm{CdS}$ e bands aretaken from ref 27 , and the $\mathrm{ZnS}$ band offsets were taken from ref 28 . In the solidstate language, photoexcitation of a semiconductor promotes an electron ( $\mathrm{e}^{-}$) from the valence band (VB) to the conduction band (CB) leaving a positive hole $\left(\mathrm{h}^{+}\right)$behind. F or small semiconductor nanoorystals, theabsoluteenergy levels of the electrons and holes can be calculated to the first approximation using the "particle in a box" model where the box has the dimensions of the nanocrystal and the charge carriers have so-called "effective masses". 29 The energy levels and wave functions shown in Figure 4 werecalculated with an extended effective mass approach taking intoaccount the Coulomb interaction of electrons and holes and finite barrier heights at the particle boundaries. ${ }^{30}$ The right side of the picture shows the

(26) Mahrt, J.; Willig, F.; Strock, W.; Weiss, D.; Kietzmann, R. Schwarzburg, K.; Tufts, B.; Trösken, B. J. Phys. Chem. 1994981888.

(27) Grätzel, M. In Photocatalysis-Fundamentals and Applications, Wiley: New York, 1989

(28) Wie, S. H.; Zunger, A. Appl. Phys. Lett. 1998, 72, 2011.

(29) Brus, L. E. J. Phys. Chem. 1986 9Q 2555. 
absolute energetic positions of the HOMO and LUMO for the diazaperylene dye which were determined by cyclic voltammetry (CV). By comparison of the molecular levels of the dye molecule with the quantized electronic levels of the semiconductor particle, it can be seen that the electroniclevels arestaggered; i.e., theHOMO andLUMO of the dye are energetically above the quantized hole and electron state of the NC, respectively. There is some uncertainty about the absolute position of the energy levels ${ }^{31}$ and band offsets which were taken from different measurements and calculations. However, the absolute energetic position of theHOMO of perylene chromophores attached to $\mathrm{TiO}_{2}$ surfaces has been determined tobe even at $5 \mathrm{eV}$ below the vacuum level. ${ }^{32}$ This observation additionally supports the assumption that the energy levels of the $\mathrm{CdSe}$ ddiazaperylene system are actually staggered, i.e., that one might expect electron transfer from the dye to the nanocrystal or hole transfer from the nanocrystal tothe dye. ${ }^{11}$ Interfacial electron transfer from the ligand to the particle is the basis of dye-sensitized solar cells $s^{33}$ and might alsohappen in this model compound if the attached dye molecules are photoexcited. This assumption is supported by the fact that partial quenching of the dye luminescence could be observed for all samples investigated. On the other hand, the fluorescence of the nanocrystals is also quenched if the NC/dye structure is photoexcited at a wavelength where only the nanocrystal and not the dye absorbs, e.g, between 500 and 600 nm for the sample shown in Figure 3. This observation can be explained by the occurrence of an effective hole transfer from the valence band state of the nanocrystal to the occupied dye level (HOMO). In addition, as this effect is not reduced by overcoating the dot with $\mathrm{ZnS}$, the hole might easily tunnel through the ZnS barrier. An alternative explanation might be the creation of interfacial electronicstates upon theattachment of the dye molecules which lead to fast nonradiative decay pathways. Timeresolved fluorescence and absorption measurements are needed to further reveal the photophysics of the NC/dye heterostructures where the tunability of the $\mathrm{CdS}$ e band gap allows for a defined adjustment of the band offsets and the ZnS shell thickness changes the tunneling barrier for the charge carriers. U sually the adjustment of interfacial energy levels is accomplished by the preparation of different compounds which alsoalters the chemical nature of the interface. As the chemical composition of the investigated $\mathrm{NC}$ /dye system remains the same, semiconductor nanocrystals with attached dye molecules arevery promising candidates for both fundamental research of charge transfer at surfaces as well as practical applications.

\section{Summary}

In summary, it is shown for the first time that supramolecular heterostructures can besynthesized from

(30) Schooss, D.; Mews, A.; Eychmüller, A.; Weller, H. Phys. Rev. B 1994, 4917072

(31) Truong, T. B. Chem. Phys. $1983,77,377$.

(32) Burfeindt, B.; Hannappel, T.; Strock, W.; Willig, F. J. Phys. Chem. 199610016463.

(33) Miller, R. J.; McLendon, G. L.; Nozik, A. J.; Schmickler, W. Willig F. In SurfaceElectron-Transfer Processes, VCH: Weinheim, 1995.

(34) Peng, X.; Wickham, J.; Alivisatos, A. P. J. Am. Chem. Soc. 1998, 1205343.
CdSesemiconductor nanocrystals and organicfluorophors. The supramolecular system consisting of $\mathrm{ZnS}$-covered $\mathrm{CdS}$ enanocrystals with a defined number of diazaperylene chromophores attached tothe particlesurface shows very interesting optical properties and serves as a model structure for interfacial electron and hole transfer. It is shown that the optical properties of the nanocrystals can be strongly influenced by the surrounding ligands, even though the particles are passivated with a material of higher band gap. Therefore, the chemical and electronic structure of theligands has tobetaken intoaccount when the particles are chemically modified for specific applications.

\section{Experimental Section}

NMR spectra wererecorded on a B ruker DRX 500 spectrometer operating at $500 \mathrm{MHz}$ for ${ }^{1} \mathrm{H}$ and $125 \mathrm{MHz}$ for ${ }^{13} \mathrm{C}$. Chemical shifts are given in ppm relativetoTMS. IR spectra were recorded on a Nicolet FT-IR 320 spectrometer. Field desorption mass spectra were recorded ona VG ZAB 2-SE -FPD mass spectrometer, acceleration voltage $8 \mathrm{kV}$. UV-vis spectra were recorded using a Bruins Instruments Omega 10 spectrometer. Fluorescence spectra were taken with a Hitachi F-4000 spectrometer. CV measurements of diazaperylene were performed in tetra- $n$ butylammonium hexafluorophosphate (TBAHF P) $\mathrm{CH}_{2} \mathrm{Cl}_{2} \mathrm{vs} \mathrm{F} \mathrm{c/}$ $\mathrm{FC}^{+}: E_{1 / 2^{\text {red }}},-1.76 \mathrm{eV} ; E_{1 / 2^{\text {ox }}}, 0.95 \mathrm{eV}$ (not reversible). Column chromatography was performed over Merck aluminum oxide 90 active basic (0.063-0.200 mm). 1,2-Dimethoxyethane was distilled from deep blue sodiumbenzophenone solution. Dimethylcadmium was vacuum distilled; diethylzinc was filtered before using. All other starting materials and reagents were obtained from commercial sources and used without further purification.

CdSe nanocrystals (1) in a size regime between 2 and $5 \mathrm{~nm}$ in diameter were synthesized accordingtoliterature ${ }^{13}$ as modified by Katari and co-workers. ${ }^{14}$ Particles larger than $5 \mathrm{~nm}$ were prepared according to the multiple injection method developed my Peng and co-workers. ${ }^{34}$ The synthesis of small $\mathrm{CdS}$ e clusters absorbing below $450 \mathrm{~nm}$ will be described in detail elsewhere. The ZnS passivation has been performed according to ref 15

1, 1'-Biisoquinoline (2) was synthesized according to the literature ${ }^{5}$

1, 12Diazaperylene (3). In a Schlenk reaction flask under a stream of purified argon, $0.5 \mathrm{~g}(1,95 \mathrm{mmol})$ of 1, 1'-biisoquindine (2) was dissolved in $8 \mathrm{~mL}$ of dry 1,2-dimethoxyethane Subsequently $1.29 \mathrm{~g}(33.1 \mathrm{mmol})$ of potassium, which was separated from its oxide layer and shredded intosmall pieces, was added. The reaction mixture was stirred under argon for $12 \mathrm{~h}$ at room temperature At the end of the reaction, the mixture showed a deepbluecol or caused by the dianion. Theremaining potassium, which conglomerated to one piece, was removed under argon and the solution was allowed to stir under a stream of dried air for additional $4 \mathrm{~h}$. After disappearance of the blue color, the solvent was evaporated and the residue was chromatographed on aluminum oxide with THF. After recrystallization from dichlormethane $0.37 \mathrm{~g}(74 \%)$ of 3 were obtained as yellow needles.

${ }^{1} \mathrm{H}$ NMR $\left(500 \mathrm{MHz}, \mathrm{C}_{2} \mathrm{D}_{2} \mathrm{Cl}_{4}\right): \delta 8.72(\mathrm{~d}, 5.5 \mathrm{~Hz}, 2 \mathrm{H}), 8.28(\mathrm{~d}$, $7.3 \mathrm{~Hz}, 2 \mathrm{H}), 7.74(\mathrm{~d}, 7.9 \mathrm{~Hz}, 2 \mathrm{H}), 7.69(\mathrm{dd}, 7.9 \mathrm{~Hz}, 7.3 \mathrm{~Hz}, 2 \mathrm{H})$, $7.61(\mathrm{~d}, 5.5 \mathrm{~Hz}, 2 \mathrm{H}) .{ }^{13} \mathrm{C}$ NMR $\left(125 \mathrm{MHz}, \mathrm{C}_{2} \mathrm{D}_{2} \mathrm{Cl}_{4}\right): \delta 151.0$, 145. 1, 137.3, 130.9, 127.7, 125.2, 122. 5, 122.0. IR spectrum(KBrPressling) $v\left(\mathrm{~cm}^{-1}\right): 3035 ; 1604 ; 1572 ; 1554 ; 1375 ; 1346 ; 1288$; 213; 1139; 833; 812; 795; 779; 757; 645; 546; 529; 465. FD-MS: $\mathrm{m} / \mathrm{z}=254.3(100 \%)\left[\mathrm{M}^{+}\right]$.

Acknowledgment. This work has been supported by the "Stiftung Rheinland-Pfalz für Innovation" under Contract No 8312-38 62 61/371. The authors thank Dr. Jochen Übe for CV measurements and Dr. Manfred Wagner for NMR measurements.

LA0016367 